\title{
Treatment of Acute Psychosis Without Neuroleptics: Two-Year Outcomes From the Soteria Project
}

\author{
JOHN R. BOLA, PH.D., ${ }^{1}$ and LOREN R. MOSHER, M.D. ${ }^{2}$
}

\begin{abstract}
The Soteria project (1971-1983) compared residential treatment in the community and minimal use of antipsychotic medication with "usual" hospital treatment for patients with early episode schizophrenia spectrum psychosis. Newly diagnosed DSM-II schizophrenia subjects were assigned consecutively (1971 to $1976, N=79$ ) or randomly (1976 to $1979, N=100$ ) to the hospital or Soteria and followed for 2 years. Admission diagnoses were subsequently converted to DSM-IV schizophrenia and schizophreniform disorder. Multivariate analyses evaluated hypotheses of equal or better outcomes in Soteria on eight individual outcome measures and a composite outcome scale in three ways: for endpoint subjects $(N=160)$, for completing subjects $(N=129)$, and for completing subjects corrected for differential attrition $(N=129)$. Endpoint subjects exhibited small to medium effect size trends favoring experimental treatment. Completing subjects had significantly better composite outcomes of a medium effect size at Soteria $(+.47 \mathrm{SD}, p=.03)$. Completing subjects with schizophrenia exhibited a large effect size benefit with Soteria treatment $(+.81$ $\mathrm{SD}, p=.02$ ), particularly in domains of psychopathology, work, and social functioning. Soteria treatment resulted in better 2-year outcomes for patients with newly diagnosed schizophrenia spectrum psychoses, particularly for completing subjects and for those with schizophrenia. In addition, only $58 \%$ of Soteria subjects received antipsychotic medications during the follow-up period, and only 19\% were continuously maintained on antipsychotic medications.
\end{abstract}

—J Nerv Ment Dis 191:219-229, 2003

It is notable that 30 years after its initial design and implementation and 17 years since completion of data collection, the Soteria project is still producing information relevant to today's management of psychosis. Soteria's original aim was to assess whether a specially designed intensive psychosocial treatment, a relationship-focused therapeutic milieu incorporating minimal use of antipsychotic medications for 6 weeks, could produce equivalent or better outcomes in treating newly diagnosed patients with schizophrenia compared with general hospital psychiatric ward treatment with antipsychotic medications. Soteria also intended to reduce the proportion

\footnotetext{
${ }^{1}$ Department of Social Work, University of Southern California, MRF-222, Los Angeles, California 90089-0411. Send reprint requests to Dr. Bola.

${ }^{2}$ Soteria Associates, San Diego, California.

This research was supported by grants from the National Institute of Mental Health.

Presented in part at the World Psychiatric Association's International Congress in Madrid, Spain, October 2, 2001.

The authors thank Leonard S. Miller, Ph.D., UC Berkeley, and Jim Mintz, Ph.D., UCLA, for their help with statistical consultations, and John M. Davis, M.D., University of Illinois, for critique of an earlier version of this manuscript.
}

of patients maintained on antipsychotic medications (thereby reducing exposure to drug-induced toxicities) and to reduce the rate at which early-episode clients became chronic users of mental health services. This study is unique in employing a relatively large sample $(N=179)$ of clients newly diagnosed with DSM-II schizophrenia (diagnoses were subsequently converted to DSM-IV schizophrenia and schizophreniform disorder) in a quasiexperimental research design comparing multiple outcomes at 2 years.

For many years, antipsychotic medications have been the treatment of choice for patients with early episode psychotic disorders (APA, 1997; Cole et al., 1966; Lehman and Steinwachs, 1998). However, prescription of conventional antipsychotics carries substantial risk of drug toxicities (Popp and Trezza, 1998) and structural brain changes (e.g., Madsen et al., 1998). While atypical antipsychotics exhibit a more benign short-term side-effect profile (Worrel et al., 2000), there has not yet been adequate time observing their effects to rule out emergence of additional long-term toxicities. For example, the recently reported association of atypical antipsychot- 
ics with diabetes mellitus (Sernyak et al., 2002) is cause for some concern.

In developing the Soteria approach to treatment, the desire to minimize medication-induced toxicities converged with three additional factors: the recognition of significant rates of recovery without drug treatment in early episode psychosis, the observation that many patients do not benefit from medications (through drug treatment resistance and noncompliance), and a valuing of interpersonal care and treatment of mentally ill patients.

Rates of recovery without medications are significant, particularly for those with early episode psychosis. For example, placebo recovery in the acute phase of the early NIMH multisite trial was approximately 37\% (Cole et al., 1964), and the placebo-treated group had fewer rehospitalizations at 1 year postdischarge (Schooler et al., 1967). Estimates of placebo response rates for patients with acute schizophrenia range from 10\% to 40\% (Davis et al., 1989; Thornley et al., 2001), with a median of $25 \%$ (Dixon et al., 1995). Long-term follow-up studies conducted prior to the widespread use of antipsychotic drugs report functional recovery rates greater than 50\% (Bleuler, 1978; Ciompi, 1980; Huber et al., 1980).

Not all psychotic patients benefit from drug treatment. Treatment resistance to conventional antipsychotic agents is estimated to be $20 \%$ to $40 \%$ (Hellewell, 1999). Noncompliance with conventional antipsychotics is estimated to be $41 \%$ to $55 \%$ (Fenton et al., 1997). Improved compliance with atypical antipsychotics is often assumed but has not yet been established (Wahlbeck et al., 2001).

"Traitement moral," a humanistic trend in the care and treatment of persons with mental illness, can be traced to Pinel's removing chains from the men in Paris' Bicetre Hospital in 1797. Following in the humanistic treatment tradition, Soteria incorporated aspects of moral treatment (Bockhoven, 1963), Sullivan's (1962) interpersonal theory and specially designed milieu at Shepard-Pratt Hospital in the 1920 s, and the "developmental crisis" notion that growth may be possible from psychosis (Laing, 1967; Menninger, 1959; Perry, 1974).

This is the first report from the entire Soteria sample using multivariate methods to test hypotheses of comparable outcomes over a 2-year period. We use two-tailed tests to evaluate hypotheses for each outcome in three ways: for endpoint subjects $(N=160)$, for completing subjects $(N=129)$, and for completing subjects adjusted for differential attrition $(N=129$; Heckman, 1979). Subsequently, we conducted tests for schizophrenia and schizophreniform subjects separately.

\section{Methods}

\section{Study Design}

The Soteria project employed a quasiexperimental treatment comparison using consecutive spaceavailable treatment assignment in the first cohort (1971 to $1976, N=79$ ) and an experimental design with random assignment in the second cohort (1976 to $1979, N=100$ ).

\section{Subjects}

Subjects were recruited from two county hospital psychiatric emergency rooms in the San Francisco Bay Area. All persons meeting the following criteria were asked to participate: initial diagnosis of schizophrenia by three independent clinicians (per DSMII); at least four of seven cardinal symptoms of schizophrenia (thinking or speech disturbances, catatonic motor behavior, paranoid ideation, hallucinations, delusional thinking other than paranoid, blunted or inappropriate emotion, disturbance of social behavior and interpersonal relations); judged in need of hospitalization; no more than one previous hospitalization for 4 weeks or less with a diagnosis of schizophrenia; aged 15 to 32 years; and not married. These criteria were intended to produce a relatively poor prognosis group, at heightened risk for a chronic course, through the exclusion of older and married patients (Strauss and Carpenter, 1978). After description of the study to the subjects, written informed consent was obtained from patients and their families, if available.

Emergency room staff psychiatrists made initial diagnoses. An independent research team trained to maintain interrater reliability (Kappa) of .80 or better on all measures made subsequent assessments. Measures were taken at entry, 72 hours (designed to screen out drug-induced psychoses), 6 weeks, 1 year, and 2 years postadmission. Most follow-up measures were face valid (e.g., work, living arrangements, rehospitalization, etc.).

The ethnicity of subjects $(N=171)$ was $80 \% \mathrm{Eu}-$ ropean American, 9\% African American, and 11\% other ethnic groups. Sixty-four percent $(N=179)$ were male and $36 \%$ were female. The mean age was 21.7 years (range, 15 to 32 years; $\mathrm{SD}=3.4 ; N=179$ ), with the average client coming from Hollinghead's (1957) lower-middle class (higher score is lower social class: class III is 28 to 43 ; mean SES score = $42.3 ; \mathrm{SD}=16.1$; range, 11 to $77 ; N=159$ ).

\section{Treatments}

Soteria provided predominantly extramedical treatment, employing a developmental crisis ap- 
proach to recovery from psychosis. Treatment involved a small, homelike, intensive, interpersonally focused therapeutic milieu with a nonprofessional staff that expected recovery and related with clients "in ways that do not result in the invalidation of the experience of madness" (Mosher and Menn, 1978a, p 716). Experimental treatment was provided at two facilities: at Soteria and a replication facility, Emanon. Antipsychotic medications were ordinarily not used during the first 6 weeks of treatment. However, there were explicit criteria for their short-term use during this period; $76 \%$ (62 of 82 ) received no antipsychotic medications during the initial 45-day period. After 6 weeks, medication prescription decisions were made at a treatment conference that included the client, staff, and the consulting psychiatrist. A manual describing Soteria treatment in greater detail has been published in German (Mosher et al., 1994).

Control facilities were well-staffed general hospital psychiatric units geared toward "rapid evaluation and placement in other parts of the county's treatment network" (Mosher and Menn, 1978a, p 717). In these units, virtually all subjects $(94 \%, 85$ of 90$)$ were treated with continuous courses of antipsychotic medication (average $700 \mathrm{mg}$ chlorpromazine equivalents per day), and nearly all were prescribed postdischarge medications. On discharge, subjects were referred to an extensive array of outpatient services.

\section{Measures}

Eight outcome measures were used: readmission to 24-hour care (yes or no), number of readmissions, days in readmission(s), a global psychopathology scale (Mosher et al., 1971; 1 to 7 , higher is more symptomatic), a global improvement scale (Mosher et al., 1971; coded 1 to 7, 1 = much improvement, $4=$ no change, $7=$ much worse), living independently or with peers (yes or no), an ordinal measure of working (none, part-time, full-time), and the social functioning subscale of the Brief Follow-up Rating (BFR; Sokis, 1970). For completing subjects $(N=129)$, observations to the 2-year follow-up evaluation were used. Endpoint analyses $(N=160)$ used observations to the last postdischarge observation.

Composite outcome scales were created for endpoint and completing subjects from the eight outcome measures by converting each to standardized (z) scores oriented with positive values for better outcomes and summing. Missing values were set to the subject's mean score on available standardized measures for $5 \%$ of missing endpoint and $8 \%$ of missing completer information. Cronbach's alpha was .77 and .74 for the endpoint scale and the com- pleter scale, respectively. Composite scales were then restandardized, allowing subsequent analyses to be interpreted in standard deviation (effect size) units (Neter et al., 1996).

DSM-II schizophrenia patients with symptoms for at least 6 months were rediagnosed with schizophrenia $(42 \%, 71$ of 169$)$ because the addition of this criterion was the primary change from the DSM-II to DSM-III and has been carried forward into the DSMIV. Subjects not meeting this criterion were rediagnosed with schizophreniform disorder (58\%, 98 of 169). A variable approximating days of antipsychotic use during the follow-up period (between the end of experimental control of medication at 45 days and the observation at 2 years) was created as the proportion of use $(0=$ no use, $.33=$ occasional use, $.67=$ frequent use, $1=$ continuous use) times the length (in days) of the observation period and summed (mean [SD] $=327.5$ [275]; range, 0 to 685).

\section{Statistical Analysis}

In main effect analyses, the influence of experimental treatment on composite outcome and on each outcome measure was estimated in three ways: for endpoint subjects $(N=160)$, for completing subjects $(N=129)$, and for completing subjects statistically adjusted for differential attrition (Soteria nonattrition is $83 \%$ [ 68 of 82 ] vs. hospital nonattrition of $63 \%$ [ 61 of 97 ]; chi-square $=8.86, d f=1$, $p=.00)$. This presents a range of plausible treatment effect estimates for each outcome. These analyses employed control variables for the proportion of subjects diagnosed with schizophrenia (47\% [32 of 68] in Soteria vs. 29\% [17 of 58] of hospital completers; chi-square $=4.15, d f=1, p=.04$ ) and for the length of time in the postdischarge follow-up period because Soteria's design allowed longer initial treatment stays (mean $=548$ postdischarge days for Soteria completers vs. 677 for hospital completers; $t=5.89, d f=128, p=.00)$.

Due to differential attrition across treatment groups, Heckman's (1979) procedure for correcting attrition bias was used in one set of treatment effect estimates for completers (Tables 1 through 3, column 4). This procedure involves three steps: estimating a probit model on nonattrition from baseline variables, calculating a function of the probability that a subject was not lost to follow-up (the inverse mills ratio), and using this function as a covariate in multivariate estimates of treatment effects. The inverse mills ratio from the probit model on nonattrition was assessed for collinearity with other control variables (Stolzenberg and Relles, 1997; schizophrenia, days in the follow-up period); none was found 
TABLE 1

Marginal Effects of Experimental Treatment on Two-Year Outcomes: Endpoint Subjects $(\mathrm{N}=160)$, Completers ( $\mathrm{N}=129)$ and Completers Corrected for Attrition ( $\mathrm{N}=129$ )

\begin{tabular}{lccc}
\hline Outcome Variable & Endpoint $^{a, c}$ & Completers $^{a, c}$ & Completers Adjusted $^{b, c}$ \\
\hline Composite outcome $^{d}$ & .17 & $.35^{*}$ & $.47^{* *}$ \\
Social functioning $^{e}$ & .20 & .18 & .08 \\
Global psychopathology $^{f}$ & .05 & $.21^{* *}$ & $.20^{* *}$ \\
Improvement in psychopathology $^{g}$ & .09 & $.17^{*}$ & $.17^{*}$ \\
Working $^{h}$ & & & .05 \\
$\quad$ Any & .01 & .04 & .08 \\
$\quad$ Full-time & .02 & $.19^{*}$ & .17 \\
Living alone or with peers $^{i}$ & $.18^{* *}$ & -.05 & $-.16^{*}$ \\
Readmission $^{j}$ & -.10 & -.44 & $-.98^{* *}$ \\
Number of readmissions $^{k}$ & -.30 & -4.6 & -23.6 \\
Days in readmission $^{l}$ & -.93 & \\
\hline
\end{tabular}

${ }^{a}$ Estimates control for schizophrenia/schizophreniform disorder and number of days between initial discharge and 2-year follow-up.

${ }^{b}$ Estimates control for the schizophrenia/schizophreniform disorder, number of days between initial discharge and 2-year follow-up, and differential attrition by treatment group.

${ }^{c}$ Significance tests are two-tailed: $* \mathrm{p}<.10, * * \mathrm{p}<.05$.

${ }^{d}$ Difference in the composite outcome for Soteria subjects (in standard deviation units).

${ }^{e}$ Difference in the probability of membership in the two best categories (having little or no psychopathology).

${ }^{f}$ Difference in the probability of membership in the two best categories (having excellent or very good improvement in psychopathology).

${ }^{g}$ Difference in the probability of the event occurring (readmission).

${ }^{h}$ Difference in the expected value (number of readmissions).

${ }^{i}$ Difference in the expected value (days in readmission).

${ }^{j}$ Difference in the probability of the event occurring (living alone or with peers).

${ }^{k}$ Difference in the probability of the events occurring (any work, full-time work).

${ }^{l}$ Difference in social functioning (on a 3 point scale).

TABLE 2

Marginal Effects of Experimental Treatment on Two-Year Outcomes for Schizophrenia Subjects: Endpoint (N = 63), Completers $(\mathrm{N}=49)$, and Completers Corrected for Attrition ( $\mathrm{N}=49)$

\begin{tabular}{lccc}
\hline Outcome Variable & Endpoint $^{a, c}$ & Completers $^{a, c}$ & Completers Adjusted $^{b, c}$ \\
\hline Composite outcome $^{d}$ & .39 & .38 & $.81^{* * *}$ \\
Social functioning $^{e}$ & $.64^{* *}$ & $.67^{* *}$ & $.59^{*}$ \\
Global psychopathology $^{f *}$ & $.34^{* *}$ & $.44^{*}$ & $.44^{* * *}$ \\
Improvement in psychopathology $^{g}$ & $.34^{* *}$ & $.49^{* *}$ & $.48^{* * *}$ \\
Working $^{h}$ & & & .31 \\
$\quad$ Any & .18 & .23 & $.20^{* * *}$ \\
$\quad$ Full-time & .13 & .27 & .28 \\
Living alone or with peers $^{i}$ & .19 & .12 & $-.21^{*}$ \\
Readmission $^{j}$ & .05 & .38 & -.92 \\
Number of readmissions $^{k}$ & .36 & 34.8 & -3.83 \\
Days in readmission $^{l}$ & 31.2 & & \\
\hline
\end{tabular}

${ }^{a}$ Estimates control for number of days between initial discharge and 2-year follow-up.

${ }^{b}$ Estimates control for number of days between initial discharge and 2-year follow-up, and differential attrition by treatment group.

${ }^{c}$ Significance tests are two-tailed: $*^{*}<.10, * * p<.05$.

${ }^{d}$ Difference in the composite outcome for Soteria treatment (in standard deviation units).

${ }^{e}$ Difference in the probability of membership in the two best categories (having little or no psychopathology).

${ }^{f}$ Difference in the probability of membership in the two best categories (having excellent or very good improvement).

${ }^{g}$ Difference in the probability of the event occurring (readmission).

${ }^{h}$ Difference in the expected value (number of readmissions).

${ }^{i}$ Difference in the expected value (days in readmission).

${ }^{j}$ Difference in the probability of the event occurring (living alone or with peers).

${ }^{k}$ Difference in the probability of the events occurring (any work, full-time work).

${ }^{l}$ Difference in social functioning (on a 3 point scale).

(the largest correlation was with schizophrenia, Pearson $r=-.06$, NS). The two-stage Heckman procedure results in a small distortion of standard error estimates through use of an estimated rather than an observed inverse mills ratio. Initial efforts to correct the standard errors resulted in only slight $p$-value changes (in the third decimal place); therefore, they were left uncorrected.

These analytic procedures were repeated separately for endpoint $(N=63)$ and completing sub- 
TABLE 3

Marginal Effects of Experimental Treatment on Two-Year Outcomes for Schizophreniform Subjects: Endpoint ( $\mathrm{N}=97)$, Completing Subjects ( $\mathrm{N}=80)$, and Completers Corrected for Attrition ( $\mathrm{N}=80)$

\begin{tabular}{|c|c|c|c|}
\hline Outcome Variable & Endpoint $^{a, c}$ & Completers $^{a, c}$ & Completers Adjusted $^{b, c}$ \\
\hline Composite outcome $^{d}$ & .19 & .33 & .34 \\
\hline Social functioning $^{e}$ & -.10 & -.15 & -.22 \\
\hline Global psychopathology ${ }^{f}$ & .03 & .08 & .07 \\
\hline Improvement in psychopathology ${ }^{g}$ & .06 & .05 & .06 \\
\hline \multicolumn{4}{|l|}{ Working $^{h}$} \\
\hline Any & -.01 & -.09 & -.09 \\
\hline Full-time & -.01 & -.08 & -.08 \\
\hline Living alone or with Peers ${ }^{i}$ & .17 & .13 & .12 \\
\hline Readmission ${ }^{j}$ & $-.19 *$ & -.16 & $-.20 *$ \\
\hline Number of readmissions ${ }^{k}$ & $-.59 *$ & $-.98 * *$ & $-1.24 * *$ \\
\hline Days in readmission ${ }^{l}$ & -16.4 & -30.0 & -41.5 \\
\hline
\end{tabular}

${ }^{a}$ Estimates control for number of days between initial discharge and 2-year follow-up.

${ }^{b}$ Estimates control for number of days between initial discharge and 2-year follow-up, and differential attrition by treatment group.

${ }^{c}$ Significance tests are two-tailed: $* \mathrm{p}<.10, * * \mathrm{p}<.05$.

${ }^{d}$ Difference in composite outcome for Soteria treatment (in standard deviation units).

${ }^{e}$ Difference in social functioning (on a 3 point scale).

${ }^{f}$ Difference in the probability of membership in the two best categories (having little or no psychopathology).

${ }^{g}$ Difference in the probability of membership in the two best categories (having excellent or very good improvement).

${ }^{h}$ Difference in the probability of the events occurring (any work, full-time work).

${ }^{i}$ Difference in the probability of the event occurring (living alone or with peers).

${ }^{j}$ Difference in the probability of the event occurring (readmission).

${ }^{k}$ Difference in the expected value (readmissions).

${ }^{l}$ Difference in the expected value (days in readmission).

jects $(N=49)$ with insidious-onset schizophrenia, and for endpoint $(N=97)$ and completing subjects $(N=80)$ with schizophreniform disorder. Subgroup analyses used the same control variables, omitting only the indicator variable for schizophrenia.

In each analysis, estimates were made with the multivariate statistical procedure appropriate for the level of measurement of the dependent variable: ordinary least squares (OLS) regression for interval measures (composite outcome scale, social functioning), a maximum likelihood probit for binary categorical variables (readmission, living independently), an ordered probit for ordered categorical variables (McKelvey and Zavoina, 1975; global psychopathology, improvement in psychopathology, working), and a classical tobit for lower truncated interval measures (Tobin's probit, Tobin, 1958; number of readmissions, days in readmission).

Treatment effects from probit models report the difference in the probability of the observed outcome (readmission, living independently) for experimental subjects. Experimental treatment estimates from ordered probability models report the difference in the combined probability of membership in the two best categories of the dependent variable. Effect estimates on work functioning are presented as the change in the probability of working full-time and as the change in the probability of working at all (working full-time plus working part-time) for experimental subjects. For truncated interval measures (number of readmissions, days in readmission), estimates represent the change in the expected value of the dependent variable associated with experimental treatment (see Breen, 1996, p 27, Eq. 2.18 for the specification). Analyses were conducted using the statistical software packages SPSS and LIMDEP (LIMited DEPendent variables; Greene, 1998).

\section{Results}

\section{Main Effects}

Main effect results for endpoint subjects $(N=160$; Table 1, column 2) indicate that experimentally treated subjects had a nonsignificant two tenths of a standard deviation better outcomes $(+.17 \mathrm{SD}, t=$ $1.07, d f=149$, NS, all statistical tests are two tailed). Experimentally treated endpoint subjects had significantly better outcomes on one of the eight outcomes: an 18\% higher probability of living alone or with peers $(+.18, z=1.94, d f=147, p=.05)$.

Results for completers, unadjusted for attrition $(N=129$; Table 1, column 3$)$, indicate that experimentally treated subjects had one third of a standard deviation better composite outcomes (statistical trend, +.35 SD, $t=1.73, d f=124, p=.09$ ), including significantly better outcomes on one of eight measures: a $21 \%$ higher probability of having no or very low psychopathology scale scores $(+.21, z=-2.53$, $d f=103, p=.01)$. 
Main effect results for completers adjusted for attrition $(N=129$; Table 1 , column 4$)$ indicate that experimentally treated subjects had nearly one half standard deviation better composite outcomes $(+.47 \mathrm{SD}, t=2.20, d f=123, p=.03)$ and significantly better outcomes on two of eight measures: a $20 \%$ higher probability of membership in the lowest two psychopathology categories $(+.20, z=-2.22$, $d f=102, p=.03$ ) and nearly one fewer readmission $(-0.98, z=-2.37, d f=123, p=.02)$.

\section{Schizophrenia Subjects}

Endpoint schizophrenia subjects $(N=63$; Table 2, column 2) had four tenths of a standard deviation better composite outcomes in Soteria (not statistically significant; +.39 SD, $t=1.42, d f=60, p=.16$ ). This includes significantly better outcomes on three of eight measures: a 34\% higher probability of having no or nearly no psychopathology $(+.34, z=-2.74$, $d f=58, p=.01$ ), a $34 \%$ higher probability of membership in the two best psychopathology improvement categories $(+.34, z=-2.16, d f=58, p=.03)$ and six tenths of a point (on a 3-point scale) better social functioning $(+.64, t=2.34, d f=45, p=.02)$.

Unadjusted for attrition, schizophrenia completers (Table 2, column 3) treated at Soteria had a nonsignificant four tenths of a standard deviation better outcome ( $+.38 \mathrm{SD}, t=1.19, d f=46$, NS) and statistically significant benefits on three of eight outcomes: a $44 \%$ higher probability of being in the lowest two psychopathology categories $(+.44, z=$ $-2.13, d f=36, p=.03$ ), a $49 \%$ higher probability of being in the best two psychopathology improvement categories ( $+.49, z=-2.75, d f=36, p=.01$ ), and two thirds of a point better social outcomes $(+.67$, $t=2.53, d f=37, p=.02$ ).

Adjusted for differential attrition, completing schizophrenia subjects ( $N=49$; Table 2 , column 4$)$ had eight tenths of a standard deviation better composite outcomes when treated at Soteria $(+.81 \mathrm{SD}$, $t=2.42, d f=45, p=.02$ ) and significantly better outcomes on four of eight measures: a $44 \%$ higher likelihood of having no or nearly no psychopathology ( $+.44, z=-2.11, d f=35, p=.04)$, a $48 \%$ higher likelihood of having excellent or very good psychopathology improvement $(+.48, z=-2.67, d f=34$, $p=.01)$, and a $40 \%$ higher probability of working $(+.40, z=2.30, d f=40, p=.02)$.

\section{Schizophreniform Subjects}

Schizophreniform endpoint subjects $(N=97$; Table 3 , column 2) had a nonsignificant two tenths of a standard deviation better outcome at Soteria $(+.19$ $\mathrm{SD}, t=.92, d f=94$, NS).

Unadjusted for attrition, completing schizophreniform subjects ( $N=80$; Table 3, column 3 ) had a nonsignificant one third standard deviation better composite outcome at Soteria $(+.33 \mathrm{SD}, t=1.28$, $d f=77, p=.20$ ) that includes one statistically significant finding, approximately one fewer readmission to 24 -hour care $(-0.98$ readmits, $z=-1.98$, $d f=74, p=.05)$.

Adjusted for attrition, completing schizophreniform subjects ( $N=80$; Table 3 , column 4 ) had a nonsignificant one third standard deviation better composite outcome at Soteria $(+.34 \mathrm{SD}, t=1.22$, $d f=76, \mathrm{NS}$ ), including significantly better outcomes on one of eight measures, an average of one and one quarter fewer readmissions $(-1.24$ readmits, $z=$ $-2.36, d f=76, p=.02$ ).

\section{Post hoc Analyses}

Post hoc analysis comparing endpoint subjects later lost to follow-up ( 9 Soteria and 22 hospital subjects) found no composite outcome differences $(-0.18$ vs. $=-0.23, t=.89, d f=29, \mathrm{NS})$, indicating that loss of a high-functioning subgroup of hospital subjects is not a plausible explanation for observed Soteria treatment benefits.

Investigating whether Soteria acted to reduce medication for all subjects or only for those not medicated during the follow-up period, nonmedicated completers (29 of 68 Soteria and 2 of 61 hospital subjects) were excluded in a comparison of medication use. This comparison found no betweengroup differences (Soteria mean $=421$ days $v s$. hospital mean $=437$ days; $t=-0.42, d f=96$, NS), indicating that experimental treatment does not reduce the duration of medication use for those receiving medications but only reduces the proportion of patients medicated.

Comparison of the proportions of Soteria-treated schizophrenia versus schizophreniform subjects not receiving antipsychotic medications during the follow-up period found no significant difference: $44 \%$ of schizophreniform (16 of 36) versus $41 \%$ of schizophrenia subjects (13 of 32) were not drug treated (chi-square $=.10, d f=1, \mathrm{NS}$ ), indicating Soteria was equally effective in reducing antipsychotic medication use in both groups.

\section{Discussion}

\section{Main Findings}

Despite some treatment crossover during the follow-up period, strikingly beneficial effects of Soteria 
treatment are still evident at the 2-year follow-up period. This is particularly notable because an earlier report of 2-year outcomes from the first cohort of this study described more modest benefits (Mosher and Menn, 1978a). These results extend and refine previous reports by including both cohorts and conducting multivariate endpoint and two completing subject analyses. Recall that previously reported (Mosher and Menn, 1978b; Mosher et al., 1995) separate cohort analyses of 6-week data showed significant and comparable symptomatic improvement for both groups despite marked differences in neuroleptic treatment.

Three sets of treatment effect estimates show a pattern of small to medium effect size benefits for Soteria that are larger for completing than for endpoint subjects. This may be partly due to completers having the full 2-year period in which to recover. The possibility that a group of higher functioning control subjects may have been lost to follow-up between endpoint and completion turns out not to be an explanation for Soteria benefits because endpoint subjects lost to follow-up had comparable outcomes in both treatment groups. However, due to higher attrition among the hospital-treated subjects, especially among hospital-treated schizophrenia subjects, effect estimates for completing subjects unadjusted for attrition are likely to contain a bias. Therefore, the third set of treatment effect estimates uses a statistical procedure developed by Nobel Laureate James Heckman (2000 in Economics) to more accurately estimate the effects of Soteria treatment on a new sample of similar clients. Adjusting for differential attrition, completing subjects treated at Soteria had nearly one half of a standard deviation better composite outcome scores than the usual treatment group (Table 1, column 4), a "medium" effect size (Cohen, 1987) that is statistically and practically significant. Soteria-treated subjects also had lower psychopathology scores and fewer readmissions than hospital-treated subjects.

When considering schizophrenia subjects separately, results indicate even more favorable outcomes in the Soteria-treated group. Adjusted for differential attrition, these subjects have significantly better composite outcomes of a large effect size (Cohen, 1987; "large" effect size $=.80$ ) despite not being more frequently medicated in Soteria than schizophreniform subjects.

\section{What Accounts for These Findings?}

These favorable findings from Soteria call for some explanation. Therefore, we examined possible explanations in three areas: analytic methodology, components of treatment, and similarities between Soteria and factors hypothesized as responsible for favorable developing country outcomes in World Health Organization studies (Jablensky et al., 1992; Leff et al., 1992).

\section{Analytic Methodology}

The more favorable results in the present analyses seem partly due to the larger sample and more contemporary statistical methods. We have noted several important variables related to outcomes and different across treatment groups (schizophrenia, length of the postdischarge follow-up period, and attrition). The contrast between these and previously reported results highlights the importance of including in statistical analyses relevant control variables that are a) scientifically related to study design (length of follow-up period; Wyatt, 1991), b) theoretically related to outcome (schizophrenia; Cohen and Cohen, 1983), or c) may affect the generalizability of results (differential attrition rates; Heckman, 1979).

To illustrate this point, control variables from the regression on composite outcome were added one at a time using completing subjects $(N=129$; Table 1 , compare with row 1 ). When only experimental treatment was included in the regression, the effect size estimate (regression coefficient, in standard deviation units) for experimental treatment was .12 and not statistically significant $(t=.66, d f=127$, $p=.51$ ). This is analogous to the commonly used, and perhaps overly simplistic, two-group $t$-test used in earlier reports. When the variable for length of the follow-up period was added, the effect size estimate for experimental treatment became $.32(t=1.63$, $d f=126, p=.11$ ). Adding the variable for schizophrenia (and its missing value indicator), the effect size estimate for experimental treatment became .35 (Table 1, row 1 , column $3 ; t=1.73, d f=124, p=$ .08). Finally, adding the indicator for the probability of nonattrition, the effect size estimate for experimental treatment became, as reported here, .47 (Table 1 , row 1 , column $4 ; t=2.20, d f=123, p=.03$ ). Thus, it appears that previous reports from Soteria have underestimated the benefits of experimental treatment through omission of important control variables. In sum, we view these multivariate analytic methods as more appropriate than previously used bivariate methods and as producing relatively unbiased estimates of the effectiveness of Soteria treatment.

\section{Treatment Components}

A number of therapeutic ingredients in Soteria treatment have been suggested by Mosher (2001) as 
likely sources of benefit, including a) the milieu, b) attitudes of staff and residents, c) quality of relationships, and d) supportive social processes.

Milieu. Differences between experimental and hospital milieus were assessed with the Moos Ward Atmosphere (WAS) and Community Oriented Program Environment Scales (COPES; Moos, 1974). Significant differences were found on 8 of 10 subscales, notably favoring the experimental milieu on involvement, support, and spontaneity (Wendt et al., 1983).

Attitudes. Soteria staff was significantly more intuitive, introverted, flexible, and tolerant than hospital staff (Hirshfeld et al., 1977). Soteria's atmosphere was imbued with the expectation that recovery from psychosis was to be expected (Mosher, 2001).

Therapeutic Relationships. Perhaps the most important therapeutic ingredient in Soteria emerged from the quality of relationships that formed, in part, because of the additional treatment time allowed. Within staff-resident relationships, an integrative context was created to promote understanding and the discovery of meaning within the subjective experience of psychosis. Residents were encouraged to acknowledge precipitating events and emotions and to discuss and eventually place them into perspective within the continuity of their life and social network.

Social Networks. The role of social networks in providing direct support and buffering stress for patients with psychotic disorders has been well documented (Buchanan, 1995). Social support has been positively correlated with favorable outcomes (Erickson et al., 1998). Psychotic patients tend also to have diminishing social support networks (Cohen and Sokolovsky, 1978). To address this deficit, the Soteria project provided a surrogate family for clients in residence, and a client-centered postdischarge social network grew up de novo. The result was peer support for community reintegration (e.g., peers helped to organize housing, education, work, and a social life) and an ongoing source of social support.

Supportive Social Processes. Social processes were influenced by a number of aspects of the program (Mosher, 2001): the creation of a family-like atmosphere, an egalitarian approach to relationships and household functioning, and an environment that respected and tolerated individual differences and autonomy.

\section{Cultural Factors}

Evident contrasts between Soteria and hospital treatment cultures brings to mind the superior outcomes in developing countries for patients with first-episode schizophrenia in World Health Organization (WHO) studies (Jablensky et al., 1992; Leff et al., 1992; Whitaker, 2002). There are many plausible similarities between Soteria and the supportive and collectivist social processes frequently hypothesized as responsible for better developing country outcomes. The second WHO study also reported a $43 \%$ lower proportion of patients maintained on antipsychotic medications in developing countries (16\% vs. developed countries, 59\%; Jablensky et al., 1992).

\section{Study Limitations}

This study has a number of limitations that restrict the validity and generalizability of these findings. One limitation arises from the inclusion of some second-episode clients (35\%, 63 of 179 had been previously hospitalized) and requiring both poor prognosis characteristics of young age and unmarried. Thus, this sample can be considered to be of somewhat poorer prognosis than one representative of only first-episode schizophrenia spectrum disorders.

Another limitation arises from the lack of explicit comparability between the rediagnosis of schizophrenia used here (DSM-II schizophrenia and an insidious onset of symptoms for 6 months or more) and a DSM-IV diagnosis of schizophrenia. DSM-IV also requires diminished functioning. However, diagnostic criteria in the Soteria study were quite rigorous, requiring agreement from three independent clinicians, and since all were deemed in need of hospitalization, impaired functioning can be assumed.

Attrition of $28 \%$ gives rise to concern for the sample's representativeness. This is accompanied by concern for a possible bias in the treatment effect estimate due to greater attrition in the hospital group (37\%) than in the experimental group (17\%). As detailed above, statistical methods to control for attrition bias in estimating treatment effects were used (Heckman, 1979), but these methods certainly do not replace missing subjects.

It is possible that the use of independent raters not blind to treatment could have introduced a measurement bias. While financial limitations precluded the use of blind reviewers, raters were independent of the project, rotated across conditions, and were trained to maintain high interrater reliability on the few measures that required rater judgment.

An additional limitation derives from the quasiexperimental nature of the study. While second cohort 
subjects were randomly assigned to treatment, first cohort subjects were assigned using a consecutive space available decision rule. This raises the question of group comparability. Although we did not find statistical evidence of between-group differences at baseline, there were clearly some differences. Differences tended to favor the hospital group, particularly with an initially lower proportion of insidious-onset schizophrenia subjects that became significantly different by follow-up evaluation (addressed via statistical control). However, similar results have been noted in comparing findings from experimental and quasiexperimental designs (Shadish and Ragsdale, 1996), especially when controlling for between-group differences.

\section{Clinical Implications}

On the whole, these data argue that a relationally focused therapeutic milieu with minimal use of antipsychotic drugs, rather than drug treatment in the hospital, should be a preferred treatment for persons newly diagnosed with schizophrenia spectrum disorder. We think that the balance of risks and benefits associated with the common practice of medicating nearly all early episodes of psychosis should be reexamined. In addition, the search, begun earlier, for treatment response subtypes in schizophrenia spectrum disorders (Carpenter and Heinrichs, 1981), particularly for patients not benefiting from antipsychotic medications, should be resumed. . $^{3,4}$

In many minds, and in clinical practice guidelines for schizophrenia (APA, 1997; Frances et al., 1996; Lehman and Steinwachs, 1998), the question of whether to administer antipsychotics for all patients with early episodes is answered affirmatively, and discussion of alternate interventions is thereby closed. We regard this closure of inquiry as premature. Current Scandinavian projects involving inhome family crisis intervention, avoiding use of hospitals and neuroleptics, and providing continuity of teams and approach over an extended period have shown highly promising results for the treatment of patients with newly diagnosed psychosis (Lehtinen et al., 2000; Cullberg et al., 2002). The Scandinavian results, Ciompi's Soteria replication (Ciompi et al., 1992, 1993), and the findings reported here indicate that, contrary to popular views, minimal use of an-

\footnotetext{
${ }^{3}$ Bola JR, Mosher LR (in press) Predicting drug-free treatment response in acute psychosis from the Soteria project. Schizophr Bull.

${ }^{4}$ Bola JR, Mosher LR (in press) Clashing ideologies or scientific discourse? Schizophr Bull.
}

tipsychotic medications combined with specially designed psychosocial intervention for patients newly identified with schizophrenia spectrum disorders is not harmful but appears to be advantageous.

In a well-known reanalysis of mostly first-episode schizophrenia spectrum studies comparing antipsychotic medications versus psychosocial or milieu treatment, Wyatt (1991) concluded: "early intervention with neuroleptics in first-break schizophrenic patients increases the likelihood of an improved longterm course" ( $p$ 325). This conclusion has contributed to enthusiasm for efforts to prevent psychosis through "early intervention" in the prodrome, often with lowdose atypical antipsychotic medications. However, most of the studies reviewed by Wyatt (1991) were of a preexperimental (mirror-image) design that did not control many threats to internal validity (Carpenter, 1997). In fact, a preponderance of the few available quasiexperimental or experimentally designed early episode studies in which one group was initially not medicated (Carpenter et al., 1977; Ciompi et al., 1992, 1993; Lehtinen et al., 2000; Mosher and Menn, 1978a; Rappaport et al., 1978; Schooler, 1967) show better long-term outcomes for the unmedicated subjects. In concert with the fuller presentation of Soteria results here, these studies suggest that specially designed psychosocial intervention combined with minimal medication use may be an effective treatment strategy for patients with early episode schizophrenia spectrum psychosis.

\section{References}

American Psychiatric Association (1997) Practice guidelines for the treatment of patients with schizophrenia. Am J Psychiatry 154:1-63.

Bleuler ME (1978) The long-term course of schizophrenic psychosis. In LC Wynne, RL Cromwell, S Matthysse (Eds), The nature of schizophrenia: New approaches to research and treatment (pp 631-636). New York: John Wiley and Sons.

Bockhoven (1963) Moral treatment in American psychiatry. New York: Springer.

Breen R (1996) Regression models: Censored, sample selected, or truncated data. Quantitative applications in the social sciences (pp 80). Beverly Hills: Sage.

Buchanan J (1995) Social support and schizophrenia: A review of the literature. Arch Psychiatr Nurs 9:68-76.

Carpenter WT, Heinrichs DW (1981) Treatment-relevant subtypes of schizophrenia. J Nerv Ment Dis 169:113-119.

Carpenter WT, McGlashan TH, Strauss JS (1977) The treatment of acute schizophrenia without drugs: An investigation of some current assumptions. Am J Psychiatry 134:14-20.

Carpenter WT (1997) The risk of medication-free research. Schizophr Bull 23:11-18.

Ciompi L (1980) Catamnestic long-term study on the course of life and aging of schizophrenics. Schizophr Bull 6:606-618.

Ciompi L, Duwalder H-P, Maier C, Aebi E, Trutsch K, Kupper Z, Rutishauser C (1992) The pilot project "Soteria Berne:" Clinical experiences and results. Br J Psychiatry 161:145-153. 
Ciompi L, Kupper Z, Aebi E, Duwalder HP, Hubschmidt T, Trutsch K, Ruitshauser C (1993) [The pilot project "Soteria Bern" in treatment of acute schizophrenia patients: II. Results of a comparative prospective follow-up period over 2 years]. Da Pilot-Projekt "Soteria Bern" zur behandlung akut schizophener: II. Ergebnisse der vergleichenden prospektiven verlaufsstudie uber zwei jahre. Nervenartz 64:440-450.

Cohen CI, Sokolovsky J (1978) Schizophrenia and social networks: Ex-patients in the inner city. Schizophr Bull 4:546560.

Cohen J (1987) Statistical power analysis for the behavioral sciences (Revised). Hillsdale, NJ: Lawrence Erlbaum Associates.

Cohen J, Cohen P (1983) Applied multiple regression/correlation analysis for the behavioral sciences (2nd ed, pp 545). Hillsdale, NJ: Lawrence Erlbaum Associates.

Cole JO, Klerman GL, Goldberg SC, NIMH PSC Group (1964) Phenothiazine treatment in acute schizophrenia. Arch Gen Psychiatry 10:246-261.

Cole JO, Goldberg SC, Davis JM (1966) Drugs in the treatment of psychosis: Controlled studies. In P. Solomon (Ed), Psychiatric drugs: Proceedings of a research conference held in Boston (pp 153-180). New York: Grune and Stratton.

Cullberg J, Levander S, Holmquist R, Mattson M, Weiselgran I-M (2002) One-year outcome in first episode psychosis patients in the Swedish Parachute Project. Acta Psychiatrica Scandinavica 106:276-285.

Davis JM, Barter JT, Kane JM (1989) Antipsychotic drugs. In HI Kaplan, BJ Sadock (Eds), Comprehensive textbook of psychiatry (pp 1591-1626). Baltimore: Williams and Wilkins.

Dixon LB, Lehman AF, Levine J (1995) Conventional antipsychotic medications for schizophrenia. Schizophr Bull 21:567577.

Erickson DH, Beiser M, Iacono WG (1998) Social support predicts 5-year outcome in first-episode schizophrenia. J Abnorm Psychol 107:681-685.

Fenton WS, Blyler CR, Heinssen RK (1997) Determinants of medication compliance in schizophrenia: Empirical and clinical findings. Schizophr Bull 23:637-651.

Frances A, Docherty JP, Kahn DA (1996) The expert consensus guidelines series: Treatment of schizophrenia. J Clin Psychiatry 57:5-58.

Greene WH (1998) LIMDEP: Version 7.0 user's manual (Revised, pp 925). Plainview, NY: Econometric Software Inc.

Heckman JJ (1979) Sample selection bias as a specification error. Econometrica 47:153-161.

Hellewell JS (1999) Treatment-resistant schizophrenia: Reviewing the options and identifying the way forward. J Clin Psychiatry 60:14-19.

Hirschfeld RMA, Matthews SM, Mosher LR, Menn AZ (1977) Being with madness: Personality characteristics of three treatment staffs. Hosp Community Psychiatry 28:267-273.

Hollingshead AB (1957) Two factor index of social position. New Haven: August Hollingshead.

Huber G, Gross G, Schuttler R, Linz M (1980) Longitudinal studies of schizophrenic patients. Schizophr Bull 6:592-605.

Jablensky A, Sartorius N, Ernberg G, Anker M, Korten A, Cooper JE, Day R, Bertelsen A (1992) Schizophrenia: Manifestations, incidence, can course in different cultures. A World Health Organization ten-country study. Psychol Med Monogr Suppl 20:1-97.

Laing RD (1967) The politics of experience. New York: Ballantine.

Leff J, Sartorius N, Jablensky A, Korton A (1992) The international pilot study of schizophrenia: Five-year follow-up findings. Psychol Med 22:131-145.

Lehman AF, Steinwachs DM (1998) The schizophrenia patient outcomes research team (PORT) treatment recommendations. Schizophr Bull 24:1-10.

Lehtinen V, Aaltonen J, Koffert T, Rakkolainen V, Syvalahti E (2000) Two-year outcome in first-episode psychosis treated according to an integrated model. Is immediate neuroleptisation always needed? Eur Psychiatry 15:312-320.
Madsen AL, Keidling N, Karle A, Esbjerg S, Hemmingsen R (1998) Neuroleptics in progressive structural brain abnormalities in psychiatric illness [Research letters]. Lancet 352:784-785.

McKelvey RD, Zavoina W (1975) A statistical model for the analysis of ordinal level dependent variables. J Math Sociol 4:103-120.

Menninger K (1959) A psychiatrist's world: The selected papers of Karl Menninger (pp 931). New York: Viking Press.

Moos RH (1974) Evaluating treatment environments: A social ecological approach. New York: John Wiley.

Mosher LR (2001) Soteria-California and its successors: Therapeutic ingredients. In L Ciompi, H Hoffmann, M Broccard (Eds), Wieso wirke Soteria?-ein ungewohnliche schizophreniebehandlung unter der lupe (Why does Soteria work-an unusual schizophrenia therapy under examination) (pp 1341). New York and Berne, Switzerland: Huber.

Mosher LR, Hendricks V, Participants SP (1994) Dabeisein: Das manual zur praxis in der Soteria (Treatment at Soteria House: A manual for the practice of interpersonal pshnomenology). In D Ford (Ed), Psychososoziale Arbeitshilfen 7. Berlin: Psychiatrie-Verlag.

Mosher LR, Menn AZ (1978a) Community residential treatment for schizophrenia: Two-year follow-up. Hosp Community Psychiatry 29:715-723.

Mosher LR, Menn AZ (1978b) Enhancing psychosocial competence in schizophrenia: Preliminary results of the Soteria project. In WE Fann, IC Carcan, A Pokorney, et al. (Eds), Phenomenology and treatment of schizophrenia (pp 371-386). Jamacia, NY: Spectrum Publications.

Mosher LR, Pollin W, Stabenau R (1971) Identical twins discordant for schizophrenia: Neurologic findings. Arch Gen Psychiatry 24:422-430.

Mosher LR, Vallone R, Menn A (1995) The treatment of acute psychosis without neuroleptics: Six week psychopathology outcome data from the Soteria project. Int J Soc Psychiatry 41:157-173.

Neter J, Kutner MH, Nachtsheim CJ, Wasserman W (1996) Applied linear statistical models (4th ed). Chicago: Irwin.

Perry JW (1974) The far side of madness (pp 177) Englewood Cliffs, NJ: Prentice-Hall.

Popp SM, Trezza GR (1998) Side effects of and reactions to psychotropic medications. In PM Kleespies (Ed), Emergencies in mental health practice (pp 279-311). New York: Guilford.

Rappaport M, Hopkins HK, Hall K, Belleza T, Silverman J (1978) Are there schizophenics for whom drugs may be unnecessary or contraindicated? Int Pharmacopsychiatry 13:100-111.

Schooler NR, Goldberg SC, Boothe H, Cole JO (1967) One year after discharge: Community adjustment of schizophrenic patients. Am J Psychiatry 123:986-995.

Sernyak MJ, Leslie DL, Alarcon RD, Losonczy MF, Rosenheck R (2002) Association of diabetes mellitus with use of atypical neuroleptics in the treatment of schizophrenia. Am J Psychiatry 159:561-566.

Shadish WR, Ragsdale K (1996) Random versus nonrandom assignment in controlled experiments: Do you get the same answer? J Consult Clin Psychol 64:1290-1305.

Sokis DA (1970) A brief follow-up rating. Compr Psychiatry 11:445-459.

Stolzenberg RM, Relles DA (1997) Tools for intuition about sample selection bias and its correction. Am Sociol Rev 62:494507.

Strauss JS, Carpenter WT (1978) The prognosis of schizophrenia: Rationale for a multidimensional concept. Schizophr Bull 4:56-67.

Sullivan HS (1962) Schizophrenia as a human process. New York: Norton.

Thornley B, Adams CE, Awad G (2001) Chlorpromazine versus placebo for schizophrenia. Cochrane Database Syst Rev 2:CD000284.

Tobin J (1958) Estimation of relationships for limited dependent variables. Econometrica 26:24-36. 
Wahlbeck K, Tuunainen A, Ahokas A, Leucht S (2001) Dropout rates in randomized antipsychotic drug trials. Psychopharmacology 155:230-233.

Wendt RJ, Mosher LR, Matthews SM, Menn AZ (1983) A comparison of two treatment environments for schizophrenia. In JG Gunderson, OA Will, LR Mosher (Eds), The principles and practices of milieu therapy (pp 17-33). New York: Jason Aronson Inc.
Whitaker R (2002) Mad in America: Bad science, bad medicine, and the enduring mistreatment of the mentally ill. Cambridge, MA: Perseus Books.

Worrel JA, Marken PA, Beckman SE, Ruehter VL (2000) Atypical antipsychotic agents: A critical review. Am J Health Syst Pharm 57:238-258.

Wyatt RJ (1991) Neuroleptics and the natural course of schizophrenia. Schizophr Bull 17:325-351. 